\title{
Characterization of the Key Region and Key Phosphorylation Sites of EcalCE1 for its Molecular Interaction with EcaH0S1 Protein in Eucalyptus camaldulensis
}

\section{Ling Cheng}

South China Agricultural University

Weihua Zhang

Guangdong Academy of Forestry

Jianlin Hu

South China Agricultural University

Ziyang Zhang

South China Agricultural University

Yan Liu

South China Agricultural University

yuanzhen lin ( $\square$ yzhlinscau@163.com )

South China Agricultural University https://orcid.org/0000-0001-9042-8016

Research article

Keywords: EcalCE1, Key Phosphorylation Sites, cold stress, Arabidopsis

Posted Date: January 22nd, 2020

DOI: https://doi.org/10.21203/rs.2.21485/v1

License: (c) (1) This work is licensed under a Creative Commons Attribution 4.0 International License.

Read Full License 


\section{Abstract}

\section{Background}

ICE1 (inducer of CBF expression 1), a MYC-like bHLH transcriptional activator, plays an important role in plant under cold stress via regulating transcriptional expression of downstream cold-responsive genes. Ubiquitination-proteasome pathway mediated by high expression of osmotically responsive gene1 (HOS1) can effectively induce the degradation of ICE1 and decrease the expression of expression of CBFs and their downstream genes under cold stress response in Arabidopsis, but the knowledge about ubiquitination regulation of ICE1 by HOS1 is still unknown in woody plants.

\section{Results}

The complete EcalCE1 gene and a new E3 ubiquitin ligase gene EcaHOS1 were amplified from the tissue culture seedlings of Eucalyptus camaldulensis. Yeast two-hybrid $(\mathrm{Y} 2 \mathrm{H})$ and $\mathrm{BiFC}$ assay results showed that EcalCE1 can interact with EcaHOS1 protein in the nucleus, and further $\mathrm{Y} 2 \mathrm{H}$ assay demonstrated that the 126-185 amino acid region at the N-terminus of EcaICE1 protein was indispensable for its interaction with EcaHOS1 protein. Moreover, we found that the amino acids at positions 143, 145, 158 and 184 within the key interaction region were the potential phosphorylation sites of EcalCE1 based on bioinformatics analysis, and that only the substitution of Serine (Ser) 158 by Alanine (Ala) blocked the protein-protein interactions between EcalCE1 and EcaHOS1 by $\mathrm{Y} 2 \mathrm{H}$ and $\beta$-galactosidase assays using site-direct mutagenesis. Overexpression of EcaICE1 and its mutations in Arabidopsis could significantly increase POD and SOD activities with a reduction for MDA content and up-regulate four cold-responsive genes ( CBF3, KIN1, COR15 and COR47A) in the transgenic lines.

\section{Conclusion}

We first reported that EcalCE1 could interact with EcaHOS1 protein in Eucalyptus, and identified Ser 158 of EcalCE1 as the key phosphorylation site for its interaction with EcaHOS1 protein.

\section{Background}

Cold stress is a major environmental factor that adversely affects plant growth and development, as well as the yield, product quality and geographic distribution of crops [1]. Plants must adjust various physiological and biochemical processes in response to cold stress by reprogramming gene expression [2]. Currently, the most well-understood cold signaling pathway is the ICE-CBF-COR transcriptional regulatory cascade $[3,4]$. CBF transcription factors (TFs) recognize the $C R T /$ dehydration-responsive element (DRE) elements in the promoters of certain cold-responsive (COR) genes and regulate their expressions and functions [5]. Inducer of CBF expression 1 (ICE1), a MYC-like bHLH transcriptional activator, acts upstream as a positive regulator of CBFs by binding to MYC recognition elements in the $C B F$ promoters in cold-responsive signaling $[4,6,7]$. In addition, $C B F$ genes also appear to be negatively regulated by ICE1 via its interactions with MYB15 [8] and HOS1 [9], while positively by ICE1 via their 
interactions with SIZ1 [10] and OST1 [11]. Recently, Li et al. reported that MPK3/MPK6 could interact with and phosphorylate ICE1, reducing the stability of ICE1 as well as its transcriptional activity, thus negatively regulating CBF expression and freezing tolerance in Arabidopsis [12]. Therefore, ICE1 is the key regulators of ICE-CBF-COR transcriptional regulatory cascades in the cold-responsive signaling pathway. ICE-like genes have been isolated and characterized in some woody plants as Populus suaveolens [13], Malus domestica [14], Vitis amurensis [15], E. camaldulensis [16], Pyrus ussuriensis [17], Poncirus trifoliata [18] and Hevea brasiliensis [19]. However, the positive and negative regulation pathway of ICE1 in relation to cold stress response still remains poorly understood in woody plants.

Eucalyptus species are the important commercial woody plants because of rapid growth, broad adaptability, and the source of wood pulp. However, even widely distributed, its plantation is mainly restricted to tropical and subtropical regions because of freezing sensitivity, especially for the commercial Eucalyptus species. Therefore, it is necessary to discover the molecular regulation mechanism of cold response and carry out genetic improvement on freezing tolerance in Eucalyptus. Although CBF genes have been isolated and characterized from Eucalyptus [20-25], the knowledge about its upstream regulator ICE1 and its positive and negative regulation pathway needs to be investigated. Our precious studies have revealed that ectopic expression of EcaICE1 from E. camaldulensis confers improved cold tolerance and the expression level of downstream genes in transgenic tobaccos [16]. Nevertheless, the factors controlling ICE1 protein stability associated with cold stress response in Eucalyptus are not clearly elucidated. In this study, we cloned the HOS1 gene EcaHOS1 from the E. camaldulensis and analyzed the protein interactions between EcaICE1 and EcaHOS1 using Bimolecular fluorescence complementation (BiFC) and Yeast two-hybrid $(\mathrm{Y} 2 \mathrm{H})$ assays. Subsequently, the key phosphorylation sites of EcaICE1 for its interaction with EcaHOS1 protein were predicted by bioinformatics and characterized by site-direct mutagenesis using $\mathrm{Y} 2 \mathrm{H}$ and assays.

\section{Results}

\section{Sequence Analysis of EcalCE1 and EcaHOS1}

The sequence of EcaICE1 gene was identical to our precious result [16]. The typical conserved domains, such as MYC-like bHLH domain, zipper structure at the C-terminus, S-rich (Serine-rich) acidic domain,

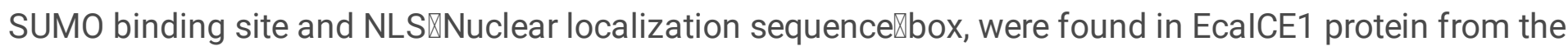
multiple alignments (Figure S1). Nevertheless, only Eucalyptus ICE1 proteins had an additional Q-rich (Glutamine-rich) domain, suggesting that the characteristics of ICE1 proteins might be different between Eucalyptus and the other plants.

The sequence of EcaHOS1 gene was 2889 bp long and encoded a complete coding frame consisting of 962 amino acids. BLAST analysis revealed that EcaHOS1 shared a high sequence identity with other plant HOS1-like proteins, such as A. thaliana (52\%, OAP11605), Vitis viinifera (60\%, NP_001268014) and Poncirus trifoliate (58\%, XP_024445010). The multiple alignments of plant HOS1 protein sequences (Fig. 1) showed that the EcaHOS1 protein had a conserved RING finger domain and ELYS domain, similar to 
other plant HOS1 proteins. The conserved RING finger domain is the crucial functional region of E3 ubiquitin ligases [26]. These results showed that EcaHOS1 was the HOS1 protein and a new E3 ubiquitin ligase from E. camaldulensis, which may have a functional role in the ubiquitination pathway.

\section{Subcellular localization of EcalCE1}

The multiple alignments (Additional file: Fig. S1) showed that there was an NLS box in the EcalCE1, implying that it may be nuclear localized protein. To confirm the result, the subcellular localization assay of EcalCE1 was carried out using a fusion protein of ECaICE1 and YFP reporter gene, driven by 35S promoter. The yellow fluorescence of EcalCE1-YFP fusion protein was detected in the nucleus (Fig. 2). These results indicated that EcalCE1 was a nuclear protein, similar to other woody plant ICE1 proteins $[17,18,19]$.

\section{EcalCE1 could interact with EcaHos1}

Previous report showed that HOS1 could interact with ICE1 and mediate the ubiquitination of ICE1 both in vitro and in vivo in $A$. thaliana, which attenuated cold stress response by the ubiquitination/proteasome pathway [9]. To elucidate whether EcaHOS1 could also interact with EcalCE1 in E. camaldulensis, a BiFC assay was performed to identify the protein-protein interactions in tobacco leaves. Microscopic visualization results (Fig. 3) revealed that there was no YFP fluorescent signal for the negative controls including EcaICE1-pSPYCE co-expressed with unfused pSPYNE or EcaHOS1-pSPYNE co-expressed with unfused pSPYCE whereas the YFP fluorescence was observed exclusively in the nucleus for the EcaICE1pSPYCE co-transfected with EcaHOS1-pSPYNE. These results showed that EcalCE1 could interact with EcaHOS1 to form heterodimers at the nucleus.

\section{Protein-protein interaction region between EcaICE1 with EcaHOS1}

We perform the $\mathrm{Y} 2 \mathrm{H}$ assay to further confirm the protein-protein interaction between EcalCE1 with EcaHOS1 and discover the key interaction region of EcalCE1 for the interaction. Unfortunately, both EcalCE1 and EcaHOS1 had autoactivation activity (Fig. S3). Then, transcriptional activation assay demonstrated that amino acids from positions 84 to 125 in EcalCE1 were critical for the transactivation activity of EcaICE1 (Fig. S2). Now the truncated EcaICE1 protein without transcriptional activation activity was constructed into vector PGBKT7, and co-transformed with AD-EcaHOS1 into yeast strain separately to find the protein-protein interaction region. The results showed that only the EcalCE1 ${ }_{\mathrm{T} 3}$ interacted with EcaHOS1 and the other regions did not (Fig. 4A), while all of these four truncated EcalCE1 proteins could interact with AtHOS1(Fig. 4B), indicating that the N-terminus region of EcalCE1(126-185aa) was the key region for its interaction with EcaHOS1 protein, quite different from $A$. thaliana [27].

\section{Effects of key phosphorylation site of EcalCE1 for its interaction with EcaHOS1 protein}

We searched the phosphorylation sites within the N-terminus region (126-185 aa) of EcalCE1 by bioinformatics software NetPhos 3.1 and found that Ser 143 (Ser at 143 aa), Thr 145(Thr at 145 aa), Ser 
158(Ser at 158 aa) and Ser 184(Ser at 184 aa) were predicted as the potential phosphorylation sites. After the substitution of these four sites by Alanine (S143A, T145A, S158A and S184A, respectively) using site-direct mutagenesis based on the EcalCE1 ${ }_{\mathrm{T} 3}$ (abbreviated as $\mathrm{T} 3$ ), $\mathrm{Y} 2 \mathrm{H}$ results (Fig. $5 \mathrm{~A}$ ) showed that only T3(S158A) blocked its interaction with EcaHOS1, while the residual three mutants and T3 still worked. It is interesting that both T3 and its mutants of EcalCE1 could interact with AtHOS1(Fig. 5B), indicating that the ubiquitination pathway of ICE1 by HOS1 may be different between Eucalyptus and Arabidopsis. $\beta$-galactosidase assay (Table 2 ) revealed that $\beta$-galactosidase activity of T3(S158A) was not significant from negative control at $P<0.05$. The $\beta$-galactosidase activity of T3(T145A) and T3(S184A) was not significant from wild type T3 at $P<0.05$, while that of T3(S143A) was significantly higher than wild type T3 $(P<0.05)$. The $\beta$-galactosidase activity assay also confirmed that only T3(S158A) blocked the protein interactions between EcalCE1 and EcaHOS1. These results suggested that Ser 158 was the key phosphorylation site of EcalCE1 for its interaction with EcaHOS1.

\section{Overexpression of EcalCE1 and its mutations in Arabidopsis}

We further transformed ECaICE1 and its mutations into Arabidopsis plants to reveal their functions. The morphological performance results (Fig. S4) showed that there was no significant difference between the phenotype of each transgenic lines and the wild type (WT) line and the ice1 mutant in Arabidopsis. After $24 \mathrm{~h}$ treatment at $4{ }^{\circ} \mathrm{C}$, physiological index measurement results (Fig. 6) showed that the activities of both POD and SOD of all five transgenic lines were significantly higher than those of WT and ice 1 mutant $(P<0.05)$, while the MDA contents were significantly lower than those of WT and ice1 mutant $(P<0.05)$. Nevertheless, the measured physiological indexes were not significantly different among these five transgenic lines. Moreover, real-time PCR results (Fig. 7) showed that the expression levels of all tested four cold-responsive genes (CBF3, KIN1, COR15 and COR47A) in the transgenic lines were significantly up-regulated $(P<0.05)$, indicating that EcalCE1 and its mutants can positively regulate downstream coldresponsive genes and may improve the stress tolerance in Arabidopsis.

\section{Discussion}

Eucalyptus can increase freezing tolerance by cold acclimation as well as other plant species. CBF genes have been cloned from Eucalyptus [20-22,25] and their overexpression in cold-sensitive Eucalyptus could improve the freezing tolerance [23]. Cao et al. further reported that there were $17 \mathrm{CBF}$ orthologs in the $E$. grandis genome and that $14 \mathrm{CBF}$ genes were located on the scaffold 1 within a cluster of about $117 \mathrm{~Kb}$ [24]. We first cloned an ICE1 gene EcalCE1 from E. camaldulensis and found that ectopic expression of EcalCE1 could confer improved cold tolerance and increase the expression level of downstream genes in transgenic tobaccos [16]. However, the factors controlling ICE1 protein stability associated with cold stress response in Eucalyptus, that is, whether there is similar ubiquitination or SUMOylation pathway of ICE1 to Arabidopsis, are still lack of empirical studies. In this current study, we further researched the ubiquitination pathway of ICE1 mediated by HOS1 in Eucalyptus and characterized EcalCE1 protein having a direct physical interaction with EcaHOS1 in vivo (Fig. 3, 4A, and 5A). 
E3-ubiquitin-ligases-mediated ubiquitination are emerging as major regulators in plants' response to abiotic stress, circadian rhythm control, cell cycling and plant-microbe interactions [28-30]. High expression of osmotically responsive genes1 (HOS1), one of the RING finger-containing E3 ubiquitin ligase, was initially described as a cold-signaling attenuator of ICE1 [9,31], and also involved in ethylene signal transduction [32] and photoperiodic flowering [33] in Arabidopsis. It is reported that most of the plant species just have a single copy of the HOS1 gene [34], suggesting that HOS1 would have important roles in various plant species. Based on the multiple alignments of EcaHOS1 with other plant HOS1 proteins (Fig. 1), the RING finger domain is highly conserved among the presented plants, and the first residue in the RING finger of all seven HOS1 proteins is Leu, which is Cys in animal inhibitor of apoptosis (IAPs) [35]. The RING-finger domain is the crucial player in the ubiquitin-dependent protein degradation system [36], indicating that plant HOS1 proteins are highly conserved E3 ubiquitin ligase. In addition, all seven HOS1 proteins have a highly conserved embryonic large molecule derived from yolk sac (ELYS) domain, which is required for the protein recruitment of the nuclear pore complex (NPC), implying that HOS1 also has nonproteolytic roles such as mRNA export and chromatin remodeling [34]. These analyses indicate that EcaHOS1 is the HOS1 gene from E. camaldulensis, and its encoded protein may have E3 ubiquitin ligase activity and mediate ubiquitination in the nucleus.

BiFC assay showed that EcalCE1 could interact with EcaHOS1 in nucleus, similar to $A$. thaliana [9], but which region of EcalCE1 was the key region for the protein-protein interactions is still unknown. Therefore, using different truncated EcalCE1 without autoactivation activity as bait protein, the N-terminal 126-185 amino acid of EcalCE1 protein was further identified as the key region by $\mathrm{Y} 2 \mathrm{H}$ assay. Interestingly, the key region of EcalCE1 (126-185 aa) is not located within the conserved bHLH domain, the zipper motif or ACTlike domain at the C-terminus region of EcalCE1. However, in Arabidopsis, AtHOS1 interacted with the Cterminus of AtICE2 containing the zipper motif [27]. Surprisingly, all truncated EcalCE1 could interact with AtHOS1, indicating that the zipper motif at C-terminus of ICE protein is indeed necessary for its interaction with AtHOS1, different from that in EcaHOS1.

A number of evidences show that non-lysine residues, such as serine, threonine and cysteine, are ubiquitylation sites of many E3 ubiquitin ligases [37-39]. Miura et al. reported that substitution of Ser 403 by Alanine (Ala) in Arabidopsis ICE1 could inhibit the polyubiquitylation of ICE1 in vivo, but did not affect the degradation of ICE1 [40]. They argued that although Ser 403 is not the main target residue for ubiquitylation or SUMOylation, it is a key residue for the attenuation of cold-stress responses by HOS1mediated degradation of ICE1 [40]. Here, we further found that Ser 143, The 145, Ser 158 and Ser 184 within 126-185 aa of EcalCE1 were predicted as the potential phosphorylation sites based on bioinformatics method. Only substitution of Ser 158 by Ala (S158A) using site-direct mutagenesis blocked the protein-protein interactions between EcalCE1 and EcaHOS1 using $\mathrm{Y} 2 \mathrm{H}$ assay (Fig. 5A), and $\beta$ galactosidase activity assay (Table 2) also confirmed this result. Surprisingly, all of EcalCE1 mutants could interact with AtHOS1 again (Fig. 5B), indicating that the conserved domains at the C-terminus of ICE are exactly essential for its interactions with AtHOS1 and that the ubiquitylation site of ICE for AtHOS1 maybe the Ser 403. 
To further elucidate the molecular function of EcalCE1 and its mutants in Arabidopsis, relative mRNA transcription levels of stress responsive genes of Arabidopsis were also measured. It is well known that overexpression of ICE and ICE-like genes can intensely induced the expression of CBF3 in plants [41]. Here, CBF3, KIN1, COR15, and COR47A were strongly expressed not only in the transgenic lines of ECaICE1, similar to our precious studies[42], but also in those of its mutants, than the WT and ice1 mutant after cold treatment (Fig. 6), showing that the site-direct mutagenesis of ECaICE1 did not affect its functions in Arabidopsis. Meanwhile, ROS-scavenging enzymes SOD, POD, and CAT are mainly involved in ROS detoxification under cold stress [43]. In our study, transgenic plants of EcalCE1 and its mutants exhibited higher activities of POD and SOD with lower MDA content than the WT and ice1 mutant after cold treatments, indicating that the site-direct mutagenesis of EcalCE1 did not affect the efficiency of ROS-scavenging system in transgenic Arabidopsis plants under cold stress.

Our results suggested that ubiquitination pathway of ICE1 mediated by HOS1 may be different between Eucalyptus and Arabidopsis and that Ser 158 was the key phosphorylation site of EcalCE1 for its interaction with EcaHOS1. As for whether EcaHOS1 acts as an E3 ubiquitin protein ligase and whether it could mediate the degradation of EcalCE1 for playing a role in cold stress response in Eucalyptus, including whether Ser 158 affects the EcaHOS1-mediated ubiquitination of ICE1, need further experiments to investigate.

\section{Conclusions}

Herein, we amplified the gene ECaICE1 and ECaHOS1 from E. camaldulensis, and the EcalCE1 sequence was the same as the previous result, and the deduced EcaHOS1 protein was highly conserved with other plant HOS1 proteins. EcalCE1 was located in nucleus, and it could interact with EcaHOS1 in nucleus by BiFC assay. Moreover, $\mathrm{Y} 2 \mathrm{H}$ assays confirmed the interaction between EcalCE1 with EcaHOS1 and revealed that the N-terminal region from position 126 to 185 in EcalCE1 was the key region for its interaction with EcaHOS1 protein. Ser 158 was the key phosphorylation site of EcalCE1 for its interaction with EcaHOS1 by $\mathrm{Y} 2 \mathrm{H}$ and $\beta$-galactosidase assay using site-direct mutagenesis. Finally, site-direct mutagenesis of EcalCE1 did not affect efficiency of ROS-scavenging system and the molecular functions of ECaICE1 in transgenic Arabidopsis plants under cold stress. Taken together, we made a foundation on the ubiquitination regulation mechanism of EcalCE1 mediated by EcaHOS1.

\section{Methods}

\section{Plant materials and treatments}

The experimental research on all plants complied with institutional, national and international guidelines. All plants were grown in a glasshouse at the Guangdong Key Laboratory for Innovative Development and Utilization of Forest Plant Germplasm, South China Agricultural University. The tissue culture plantlets of 
E. camaldulensis cv. 103 (a gift from Dr. Qian Zhang, Guangdong Academy of Forestry, China) were performed as described previously [16] and 30-day-old rooting plantlets were used in this study. No permission was needed for obtaining and using the tissue culture plantlets of E. camaldulensis cv. 103 .

Tobacco (Nicotiana benthamiana, a gift from Prof. Aimin Wu, South China Agricultural University, China) plants were cultured in a growth chamber at $25^{\circ} \mathrm{C}$ with $16 / 8 \mathrm{~h} \mathrm{light/dark} \mathrm{photoperiod,} \mathrm{and} \mathrm{5-week-old}$ plants were used for further subcellular localization and BiFC analysis.

The A. thaliana wild type (Col-0) used as the control group was provided by Prof. Aimin Wu from South China Agricultural University. The AtICE1 knockout mutants (ice1, SALK_003426) were purchased from The Arabidopsis Information Resource (TAIR; http://www.arabidopsis.org/). Arabidopsis plants were cultured at $23^{\circ} \mathrm{C}$ with $16 / 8 \mathrm{~h}$ light/dark photoperiod.

\section{RNA isolation, gene cloning and sequence analysis}

Total RNA was extracted as described previously [16], and treated with DNase I (Promega, Madison, WI, USA). $1 \mu \mathrm{g}$ DNA-free total RNA was used the template for synthesizing the first strand cDNA (PrimeScript II 1st Strand cDNA Synthesis Kit; Takara, Dalian, China). The primers of EcalCE1 and EcaHOS1 (listed as table 1) were used for amplifying the aim genes with the first strand CDNA as the template. The aim genes were sequenced at Beijing genomics Institute (BGI, China). The coding sequence (CDS) of EcalCE1 and EcaHOS1 were predicted using FGENESH 2.6 software ( http://linux1.softberry.com/berry.phtml? topic=fgenesh\&group=programs\&subgroup=gfind ), and further confirmed by BLASTP program on the NCBI website (http://blast.ncbi.nlm.nih.gov/Blast.cgi). The protein secondary domains were predicted by Motif scan (https://myhits.isb-sib.ch/cgi-bin/motif_scan). Finally, sequence alignments with other plants were performed with CLUSTALX software.

\section{Subcellular localization}

The full-length CDS of EcaICE1 was amplified by RT-PCR using primers (listed as Supplemental table S1) and fused into the 5 -terminus of the yellow fluorescent protein (YFP) of the pEarleyGate101 vector, driven by CaMV 35S promoter. The recombinant plasmid 35S.:EcaICE1-YFP was transferred into Agrobacterium tumefaciens strain GV3101 by heat shock method. The strain GV3101 harboring plasmid 35S::EcalCE1YFP were cultured in the liquid yeast extract broth medium at $28^{\circ} \mathrm{C}$ on a shaker at $220 \mathrm{rpm}$ until the absorbance of the OD measurement at $660 \mathrm{~nm}$ reached 1.0 1.5, and then the culture were centrifuged at $8000 \times \mathrm{g}$ for $5 \mathrm{~min}$. The thallus was mixed with the infecting solution containing $1 \% \mathrm{MES}, 1 \% \mathrm{MgCl}_{2}$ and $0.1 \%$ acetosyringone, and cultured for $3 \mathrm{~h}$ at $28^{\circ} \mathrm{C}$ and then injected into the abaxial surfaces of 5-weekold tobacco leaves via a syringe with an incubator for 48-72 h. The YFP fluorescence in the tobacco leaves was visualized using a confocal microscopy (Zeiss LSM 710, Carl, Germany) with excitation and emission at 513 and $527 \mathrm{~nm}$, respectively.

\section{Bimolecular fluorescence complementation (BiFC) assay}


To perform BiFC assays, the whole CDS of EcaICE1 and EcaHOS1 (without their stop codons) were subcloned into pUC-pSPYNE or pUC-pSPYCE vectors as described previously [44] using primers (listed as Supplemental table S2). Expressions of each target gene alone were used as negative controls. The recombinant vectors were used for transient assays of tobacco leaves as described earlier. The transformed tobacco leaves were then kept in an incubator at $22{ }^{\circ} \mathrm{C}$ for $24-48 \mathrm{~h}$. YFP signal was examined using a confocal microscope (Zeiss LSM 710, Carl, Germany).

\section{Yeast two-hybrid (Y2H) assay}

Yeast two-hybrid assays were carried out using the Matchmaker ${ }^{\text {TM }}$ gold Yeast two-Hybrid Systems (Clontech, USA). Different truncated CDS of ECaICE1 without transcriptional activation activity were subcloned into pGBKT7 (BD) to form the bait vector. The full-length CDS of EcaHOS1 was amplified and inserted into pGADT7 (AD) to construct the prey vector (AD-EcaHOS1). The primers are listed in Supplemental table S3. The bait and prey vectors were co-transformed into yeast strain gold $\mathrm{Y} 2 \mathrm{H}$ using lithium acetate ( $\mathrm{LiAC}$ ) method. Then yeast cells were plated on SD/-LW medium (minimal media double dropouts, SD basal medium without Leu and Trp) according to the manufacturer's protocol (Clontech, USA) for $72 \mathrm{~h}$. Transformed colonies were sprayed on SD/-LWHA medium (minimal media quadruple dropouts, SD medium with -Leu/-Trp/-Ade/-His) containing 125- $\mu \mathrm{M}$ Aureobasidin A (AbA), to test for possible protein-protein interactions, according to the yeast cell growth status. Each experiment replicated three technological repeats in separate experiments.

\section{Phosphorylation sites prediction and site-direct mutagenesis}

The phosphorylation sites within the key region of EcalCE1 for its interaction with EcaHOS1 were predicted using NetPhos 3.1 server ( http://www.cbs.dtu.dk/services/NetPhos/ ), and further analyzed by ProtParam program on the expasy website (http://web.expasy.org/protparam). The potential phosphorylation sites were used for substitution into alanine by site-direct mutagenesis. Site-direct mutagenesis experiments were carried out with KOD-Plus Mutagenesis Kit (TOYOBOCO, China). The BD${ }_{\text {EcalCE}}{ }_{\mathrm{T} 3}$ plasmid was used as the temple for the site-saturation mutagenesis. The primers were listed in Supporting Information Table S4. All mutants were confirmed by sequencing at Beijing Genomics Institute (BGI, China). The BD-EcalCE1 ${ }_{\mathrm{T} 3}$ and its mutants were used for further $\mathrm{Y} 2 \mathrm{H}$ and $\beta$-galactosidase activity assays.

\section{Assay of $\beta$-galactosidase activity}

The $\beta$-galactosidase activity was measured based on protocols from the yeast $\beta$-galactosidase assay kit manual (Thermo, USA). Single yeast colonies grown on SD/-LW medium of BD-EcalCE $1_{\mathrm{T} 3}$ and its mutants with AD-EcaHOS1 were picked and transferred into $5 \mathrm{~mL}$ YPDA liquid medium, and incubated at $30{ }^{\circ} \mathrm{C}$ with $200 \mathrm{rpm}$ shaker for 10-14 h, and the OD measurement at $660 \mathrm{~nm}$ was recorded. $1.0 \mathrm{~mL}$ of the culture medium was centrifuged at $13,000 \mathrm{~g}$ for $1 \mathrm{~min}$, and the supernatant was removed. Then, $250 \mu \mathrm{L}$ Y-PER and $250 \mu \mathrm{L}$ ONPG solution were added, and immediately incubated at $37^{\circ} \mathrm{C}$. When the mixed solution turned yellow, $200 \mu \mathrm{L}$ stop solution was added to stop the reaction, and recorded the reaction time. After 
centrifugation at $13,000 \mathrm{~g}$ for $30 \mathrm{~s}, 200 \mu \mathrm{L}$ supernatant was measured for the OD measurement at 420 $\mathrm{nm}$. Each assay replicated three technological repeats in separate experiments. $\beta$-galactosidase activity was calculated as follows:

$\beta$-Galactosidase $($ units $)=\left(1000 \times \mathrm{OD}_{420}\right)\left(\mathrm{T} \times \mathrm{V} \times \mathrm{OD}_{660}\right)$

where $\mathrm{OD}_{420}, \mathrm{~T}, \mathrm{~V}$, and $\mathrm{OD}_{660}$ were the $\mathrm{OD}$ measurement at $420 \mathrm{~nm}$, reaction time (min), reaction solution volume $(\mathrm{mL})$, and the OD measurement at $660 \mathrm{~nm}$, respectively.

\section{Plasmid construction and plant transformation}

The coding regions of EcalCE1 and its mutants were cloned into the binary vector pEarleyGate 101 driven CaMV 35 S promoter. The constructed plasmids were transformed into the Arabidopsis through floral dipping method [45] using Agrobacterium tumefaciens GV3101, respectively. The transgenic lines were obtained by antibiotic selection and confirmed by PCR and qRT-PCR analyses. Homozygous T4 generation plants from transgenic lines were used for subsequent experiments.

\section{RNA isolation and qRT-PCR analysis}

Total RNAs were extracted from Arabidopsis by using plant RNA Kit (OMEGA, Beijing, China). qRT-PCR was performed on a Bio-Rad CFX96TM real-time thermal PCR system (Bio-Rad, Hercules, CA, USA) by using Takara SYBR Premix EX Taq mix kit (Takara, Japan) according to the manufacturer's instructions. The relative expression level was calculated by method [46]. Results were obtained using three biological replicates from each sample. Four genes (CBF3, COR15A, COR47 and KIN1) were used for gene expression analysis and actin was used as reference gene for Arabidopsis. The primers used for the qRTPCR are listed in Table S1.

\section{Measurements of POD, SOD and MDA}

Two-month-old tobacco plantlets were cold-acclimated at $15 \mathrm{C}$ for 3 days, and then treated at $4 \mathrm{C}$ for 1,3 , or 5 days. $1 \mathrm{~g}$ leaves were ground in extraction buffer to determine SOD, and POD activities and MDA content described previously [42]. One unit of SOD activity was defined as the amount of enzyme inhibiting photochemical reduction of Nitrotetrazolium Blue chloride (NBT) by $50 \%$. One unit of POD activity was defined as the amount of enzyme causing a 0.01 increase in $\mathrm{OD}_{470}$ per min under assay conditions. MDA content was measured by the thiobarbituric acid reaction. Results were obtained using three biological replicates from each sample.

\section{Data statistical analysis}

The data values presented were the means \pm standard errors (SE) of three replicates through statistical analysis via $\mathrm{R}$ software ( $\mathrm{v}$ 3.5.1), and further analyzed by ANOVA and Duncan's multiple range test to compare the differences between treatments at the $P<0.05$ level. 


\section{Declarations}

\section{Acknowledgements}

Prof. Aimin Wu (South China Agricultural University) is thanked for supplying the vectors plasmid. We also thank the Guangdong Key Laboratory for Innovative Development and Utilization of Forest Plant Germplasm for supporting plant cultivation.

\section{Authors' contributions}

$\mathrm{LC}$ and $\mathrm{WZ}$ constructed vectors and performed $\mathrm{Y} 2 \mathrm{H}$ and BiFC assays. $\mathrm{JH}$ and $\mathrm{ZZ}$ did the genetic transformation and measured the physiological indexes. JH and YL did qRT-PCR assay. YL designed the project and oversaw the project management. LC, WZ, and YL wrote the manuscript. All authors contributed to project conception and data analysis. All authors have read and approved the final version of the manuscript.

\section{Funding}

This research was supported by the National Natural Science Foundation of China (No.31470673).

\section{Availability of data and materials}

The data sets supporting the results of this article are included within the article and its additional files.

\section{Ethics approval and consent to participate}

The experimental research on all plants complied with institutional, national and international guidelines. The researchers only collected the tissue culture plantlets of $E$. camaldulensis from the cultivated variety 103 developed by Guangdong Academy of Forestry and didn't harm the tree natural resources. The collected behavior through the consent and supervision of the local protection agency and the forestry bureau. In this study, we get the help from Guangdong Academy of Forestry. Hereby declare.

\section{Consent for publication}

Not applicable.

\section{Competing interests}

The authors declare that they have no competing interests.

\section{References}

1. Knight MR, Knight H. Low-temperature perception leading to gene expression and cold tolerance in higher plants. New Phytol. 2012; 195:737-51. 
2. Chinnusamy V, Zhu J, Zhu JK. Cold stress regulation of gene expression in plants. Trends Plant Sci. 2007; 12:444-51.

3. Thomashow MF. Plant cold acclimation: freezing tolerance genes and regulatory mechanisms. Annu Rev Plant Physiol Plant Mol Biol. 1999; 50:571-99.

4. Chinnusamy V, Ohta M, Kanrar S, Lee BH, Hong XH, Agarwal M, Zhu JK. ICE1: a regulator of coldinduced transcriptome and freezing tolerance in Arabidopsis. Gene Dev. 2003; 17:1043-54.

5. Liu Q, Kasuga M, Sakuma Y, Abe H, Miura S, Yamaguchi-Shinozaki K, Shinozaki K. Two transcription factors, DREB1 and DREB2, with an EREBP/AP2 DNA binding domain separate two cellular signal transduction pathways in drought- and low-temperature-responsive gene expression, respectively, in Arabidopsis. Plant Cell. 1998, 10:1391-406.

6. Fursova OV, Pogorelko GV, Tarasov VA. Identification of ICE2, a gene involved in cold acclimation which determines freezing tolerance in Arabidopsis thaliana. Gene. 2009; 429:98-103.

7. Chinnusamy V, Zhu JK, Sunkar R. Gene regulation during cold stress acclimation in plants. Methods Mol Biol. 2010; 639:39-55.

8. Agarwal M, Hao Y, Kapoor A, Dong C, Fujii H, Zheng X, Zhu JK. A R2R3 Type MYB transcription factor is involved in the cold regulation of CBF genes and in acquired freezing tolerance. J Biol Chem. 2006; 281:37636-45.

9. Dong $\mathrm{CH}$, Agarwal M, Zhang YY, Xie Q, Zhu JK. The negative regulator of plant cold responses, HOS1, is a RING E3 ligase that mediates the ubiquitination and degradation of ICE1. PNAS. 2006; 103:82816.

10. Miura K, Jin JB, Lee J, Yoo CY, Stirm V, Miura T, Ashworth EN, Bressan RA, Yun DJ, Hasegawa PM. SIZ1-mediated SUMOylation of ICE1 controls CBF3/DREB1A expression and freezing tolerance in Arabidopsis. Plant Cell. 2007; 19:1403-14.

11. Ding YL, Li H, Zhang XY, Xie Q, Gong ZZ, Yang SH. OST1 kinase modulates freezing tolerance by enhancing ICE1 stability in Arabidopsis. Dev Cell. 2015; 32:278-89.

12. Li H, Ding YL, Shi YT, Zhang XY, Yang SH. MPK3- and MPK6-mediated ICE1 phosphorylation negatively regulates ICE1 stability and freezing tolerance in Arabidopsis. Dev Cell. 2017; 43:630-42.

13. Lin YZ, Zhang ZY, Liu CX, Zhu BQ, Chen XY. In silico cloning and analysis of cold-resistant transcription factor ICE1 from Populus suaveolens. Mol Plant breeding. 2007; 5:424-30. (in Chinese)

14. Feng XM, Zhao Q, Zhao LL, Qiao Y, Xie XB, Li HF, Yao YX, You CX, Hao YJ. The cold-induced basic helix-loop-helix transcription factor gene $\mathrm{MdClbHLH1}$ encodes an ICE-like protein in apple. BMC Plant Biol. 2012; 12:22.

15. Xu W, Jiao Y, Li R, Zhang N, Xiao D, Ding X, Wang Z. Chinese wild-growing Vitis amurensis ICE1 and ICE2 encode MYC-type bHLH transcription activators that regulate cold tolerance in Arabidopsis. PLoS ONE. 2014; 9(7):e102303.

16. Lin YZ, Zheng HQ, Zhang Q, Liu CX, Zhang ZY. Functional profiling of EcalCE1 transcription factor gene from Eucalyptus camaldulensis involved in cold response in tobacco plants. J Plant Biochem Biotechn. 2014; 23(2):141-50. 
17. Huang X, Li K, Jin C, Zhang S. ICE1 of Pyrus ussuriensis functions in cold tolerance by enhancing PUDREBa transcriptional levels through interacting with PuHHP1. Sci Rep. 2015; 5:17620.

18. Huang XS, Zhang Q, Zhu D, Fu X, Wang M, Zhang Q, Moriguchi T, Liu JH. ICE1 of Poncirus trifoliata functions in cold tolerance by modulating polyamine levels through interacting with arginine decarboxylase. J Exp Bot. 2015; 66:3259-74.

19. Deng XM, Wang JX, Li Y, Wang J, Tian W. Characterization of a cold responsive HbICE1 gene from rubber trees. Trees. 2017; 31:137-47.

20. Kayal W, Navarro M, Marque G, Keller G, Marque C, Teulières C. Expression profile of CBF-like transcriptional factor genes from Eucalyptus in response to cold. J Exp Bot. 2006; 57(10):2455-69.

21. Gamboa MC, Rasmussen-Poblete S, Valenzuela PD, Krauskopf E. Isolation and characterization of a cDNA encoding a CBF transcription factor from globules. Plant Physiol Biochem. 2007; 45:1-5.

22. Navarro M, Marque G, Ayax C, Keller G, Borges JP, Marque C, Teulières C. Complementary regulation of four Eucalyptus CBF genes under various cold conditions. J Exp Bot. 2009; 60(9):2713-24.

23. Navarro M, Ayax C, Martinez Y, Laur J, Teulières C. Two EguCBF1 genes overexpressed in Eucalyptus display a different impact on stress tolerance and plant development. Plant Biotechnol J. 2011; 9(1):50-63.

24. Cao PB, Azar S, SanClemente H, Mounet F, Dunand C, Marque G, Marque C, Teulières C. Genome-wide analysis of the AP2/ERF family in Eucalyptus grandis: an intriguing over-representation of stressresponsive DREB1/CBF PLoS ONE 2015; 10(4):e0121041.

25. Nguyen HC, Cao PB, SanClemente H, Ployet R, Mounet F, Ladouce N, Harvengt L, Marque C, Teulières Special trends in CBF and DREB2 groups in Eucalyptus gunnii vs Eucalyptus grandis suggest that CBF are master players in the trade-off between growth and stress resistance. Physiol Plant. 2017; 159:445-67.

26. Stone SL, Hauksdóttir H, Troy A, Herschleb J, Kraft E, Callis J. Functional analysis of the RING-type ubiquitin ligase family of Arabidopsis. Plant Physiol. 2005; 137(1):13-30.

27. Kim YS, Lee M, Lee J, Lee H, Park C. The unified ICE-CBF pathway provides a transcriptional feedback control of freezing tolerance during cold acclimation in Arabidopsis. Plant Mol Biol. 2015; 89:187-201.

28. Hervé C, Lefebvre B, Cullimore J. How many E3 ubiquitin ligase are involved in the regulation of nodulation. Plant Signal Behav. 2011; 6(5):660-4.

29. Liu JJ, Xia ZL, Wang, MP, Zhang XQ, Yang TZ, Wu JY. Overexpression of a maize E3 ubiquitin ligase gene enhances drought tolerance through regulating stomatal aperture and antioxidant system in transgenic tobacco. Plant Physiol Biochem. 2013; 73:114-20.

30. Ling QH, Jarvis P. Regulation of chloroplast protein import by the ubiquitin E3 ligase SP1 is important for stress tolerance in plants. Curr Biol. 2015; 25(19):2527-34.

31. Lee H, Xiong LM, Gong ZZ, Ishitani M, Becky S, Zhu JK. The Arabidopsis HOS1 gene negatively regulates cold signal transduction and encodes a RING finger protein that displays cold-regulated nucleo-cytoplasmic partitioning. Gene Dev. 2001; 15(7):912-24. 
32. Lee K, Seo PJ. The E3 ubiquitin ligase HOS1 is involved in ethylene regulation of leaf expansion in Arabidopsis. Plant Signal Behav. 2015; 10(3):e1003755.

33. Lazaro A, Valverde F, Jarillo MP. The Arabidopsis E3 ubiquitin ligase HOS1 negatively regulates CONSTANS abundance in the photoperiodic control of flowering. Plant Cell. 2012; 24:982-99.

34. Jung JH, Lee HJ, Park MJ, Park CM. Beyond ubiquitination: proteolytic and nonproteolytic roles of HOS1. Trends Plant Sci. 2014; 19:538-45.

35. Miller LK. An exegesis of IAPs: Salvation and surprises from BIR motifs. Trends Cell Biol. 1999; 9:323-8.

36. Hong JK, Choi HW, Hwang IS, Hwang BK. Role of a novel pathogen-induced pepper C3-H-C4 type RING-finger protein gene, CaRFP1, in disease susceptibility and osmotic stress tolerance. Plant Mol Biol. 2007; 63(4):571-88.

37. Cadwell K, Coscoy L. Ubiquitination on nonlysine residues by a viral E3 ubiquitin ligase. Science. 2005; 309:127-30.

38. Tait SW, deVries E, Maas C, Keller AM, DSantos CS, Borst J. Apoptosis induction by Bid requires unconventional ubiquitination and degradation of its $\mathrm{N}$-terminal fragment. J Cell Biol. 2007;179:1453-66.

39. Wang X, Herr RA, Chua WJ, Lybarger L, Wiertz EJ, Hansen TH. Ubiquitination of serine, threonine, or lysine residues on the cytoplasmic tail can induce ERAD of MHC-I by viral E3 ligase mK3. J Cell Biol. 2007; 177:613-24.

40. Miura K, Ohta M, Nakazawa M, Ono M, Hasegawa PM. ICE1 Ser403 is necessary for protein stabilization and regulation of cold signaling and tolerance. Plant J. 2011; 67(2):269-79.

41. Miura K, Furumoto T. Cold signaling and cold response in plants. Int J Mol Sci. 2013;14:5312-5337.

42. Lin YZ, Lin, SZ, Guo H, Zhang ZY, Chen XY. Functional analysis of PsG6PDH, a cytosolic glucose-6phosphate dehydrogenase gene from Populus suaveolens, and its contribution to cold tolerance improvement in tobacco plants. Letters. 2013;35(9):1509-18.

43. Miller G, Suzuki N, Ciftci-Yilmaz S, Mittler R. Reactive oxygen species homeostasis and signalling during drought and salinity stresses. Plant Cell Environ. 2010; 33:453-467.

44. Walter M, Chaban C, Schütze K, Batistic O, Weckermann K, Näke C, Blazevic D, Grefen C, Schumacher $\mathrm{K}$, Oecking $\mathrm{C}$, Harter K, Kudla J. Visualization of protein interactions in living plant cells using bimolecular fluorescence complementation. Plant J. 2004; 40(3):428-38.

45. Clough SJ, Bent AF. Floral dip: a simplified method for agrobacterium-mediated transformation of Arabidopsis thaliana. Plant J. 1999;16(6):735-43.

46. Livak KJ, Schmittgen TD. Analysis of relative gene expression data using real-time Quantitative PCR and the Methods. 2001;25, 402-8.

\section{Tables}

Table 1 Primers used in the experiments 


\begin{tabular}{ll}
\hline Primers & Primer sequences $\left(5^{\prime} \rightarrow 3^{\prime}\right)$ \\
\hline EcaICE1-F & ATGGTTCTGGGTCCCAGCG \\
EcaICE1-R & TCACACCATCCCATGGAAGC \\
EcaHOS1-F & ATGGAGGCGAGCGGCTG \\
EcaHOS1-R & TCATCGTCTGGCGAATCTGCTT \\
\hline
\end{tabular}

Table 2 Measurement of $\beta$-galactosidase activity of protein interactions of EcaICE1-T3 and its mutants with EcaHOS1

\begin{tabular}{lllll}
\hline BD vector & AD vector & $\begin{array}{l}\text { Yeast cells } \\
\text { on SD/-LW }\end{array}$ & $\begin{array}{l}\text { Yeast cells } \\
\text { on SD/-LWHA }\end{array}$ & $\beta$-galactosidase activity \\
\hline T3(S143A) & EcaHOS1 & White colonies & White colonies & $27.29 \pm 2.72 \mathrm{a}$ \\
T3(T145A) & EcaHOS1 & White colonies & White colonies & $13.29 \pm 0.31 \mathrm{~b}$ \\
\hline T3(S158A) & EcaHOS1 & White colonies & None & $5.90 \pm 1.74 \mathrm{c}$ \\
\hline T3(S184A) & EcaHOS1 & White colonies & White colonies & $13.56 \pm 0.15 \mathrm{~b}$ \\
T3 & EcaHOS1 & White colonies & White colonies & $12.32 \pm 0.16 \mathrm{~b}$ \\
BD & $\mathrm{AD}$ & White colonies & None & $5.16 \pm 1.35 \mathrm{c}$ \\
\hline
\end{tabular}

Note: Values for $\beta$-galactosidase activity followed by the same letter are not significantly different at $\alpha=0.05$ using Duncan's test.

BD: pGBKT7 vetor; AD: pGADT7 vetor; T3: truncated EcaICE1-T3; T3(S143A), T3(T145A), T3(S158A), T3(S184A): the mutant of truncated EcaICE1-T3 at serine 143, threonine 154, serine 158 and serine 184 by alanine, respectively; SD/LW: SD/-Leu-Trp: double dropouts, SD medium with -Leu/-Trp; SD/-LWHA: quadruple dropouts, SD medium with-LeuTrp-His-Ade.

\section{Figures}

\section{Figure 1}

Amino acid alignment of the EcaHOS1 protein with six plant HOS1 proteins The predicted protein domains were shown. The Arabidopsis thaliana AtHOS1 (Accession: AAP14668), E. camaldulensis EcaHOS1 (Accession: MH899181), E. grandis EgrHOS1 (Accession: XP_010055472), Citrus trifoliata CtrHOS1 (Accession: ACY92092), Populus trichocarpa PtrHOS1 (Accession: XP_024445010), Prunus persica PpeHOS1 (Accession: XP_020419310), Theobroma cacao TcaHOS1 (Accession: EOY24269) and Vitis vinifera VvHOS1 (Accession: NP_001268014) proteins are included. 
Bright field

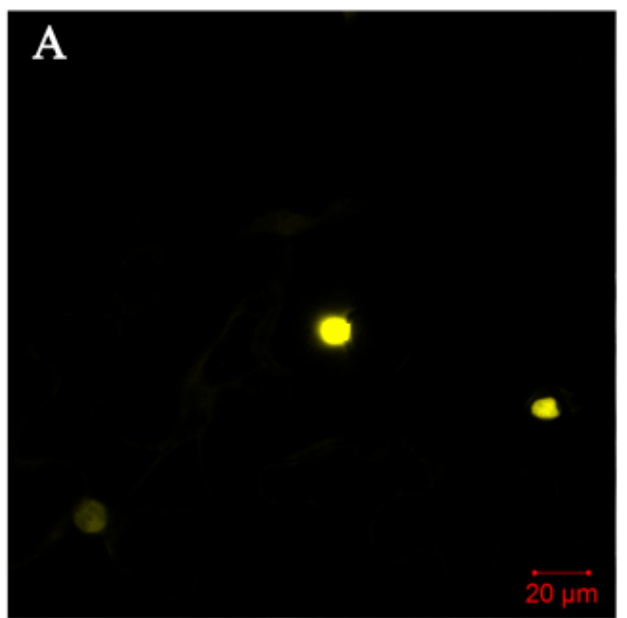

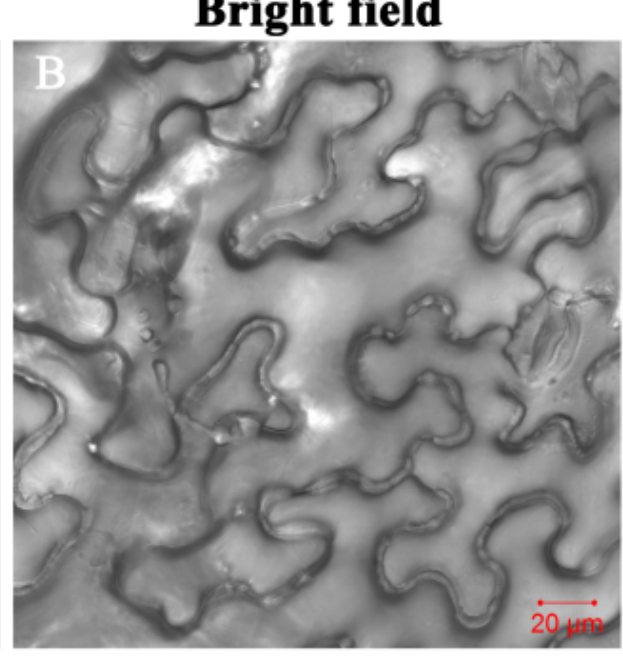

Merge

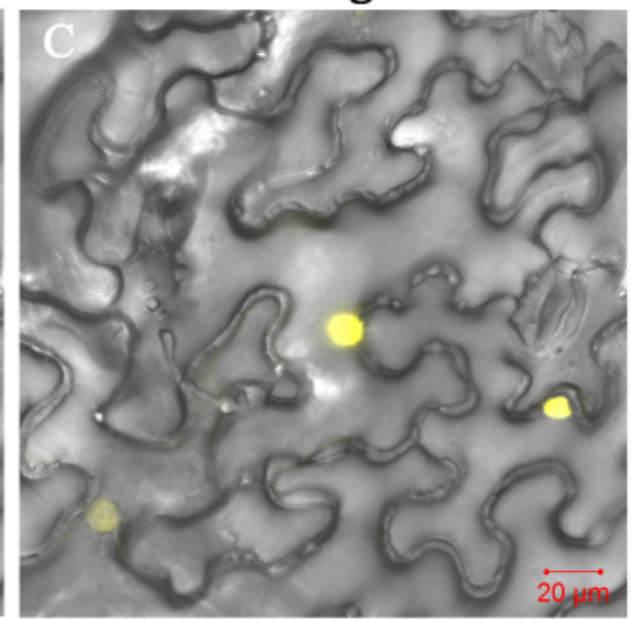

Figure 2

Subcellular localization of EcaICE1 A: the fluorescence signal plot of EcaICE1 in the dark field, B: the plot of EcalCE1 in the bright field, C: the plot of dark field and bright field superposition. Bar=20 $\mu \mathrm{m}$. 

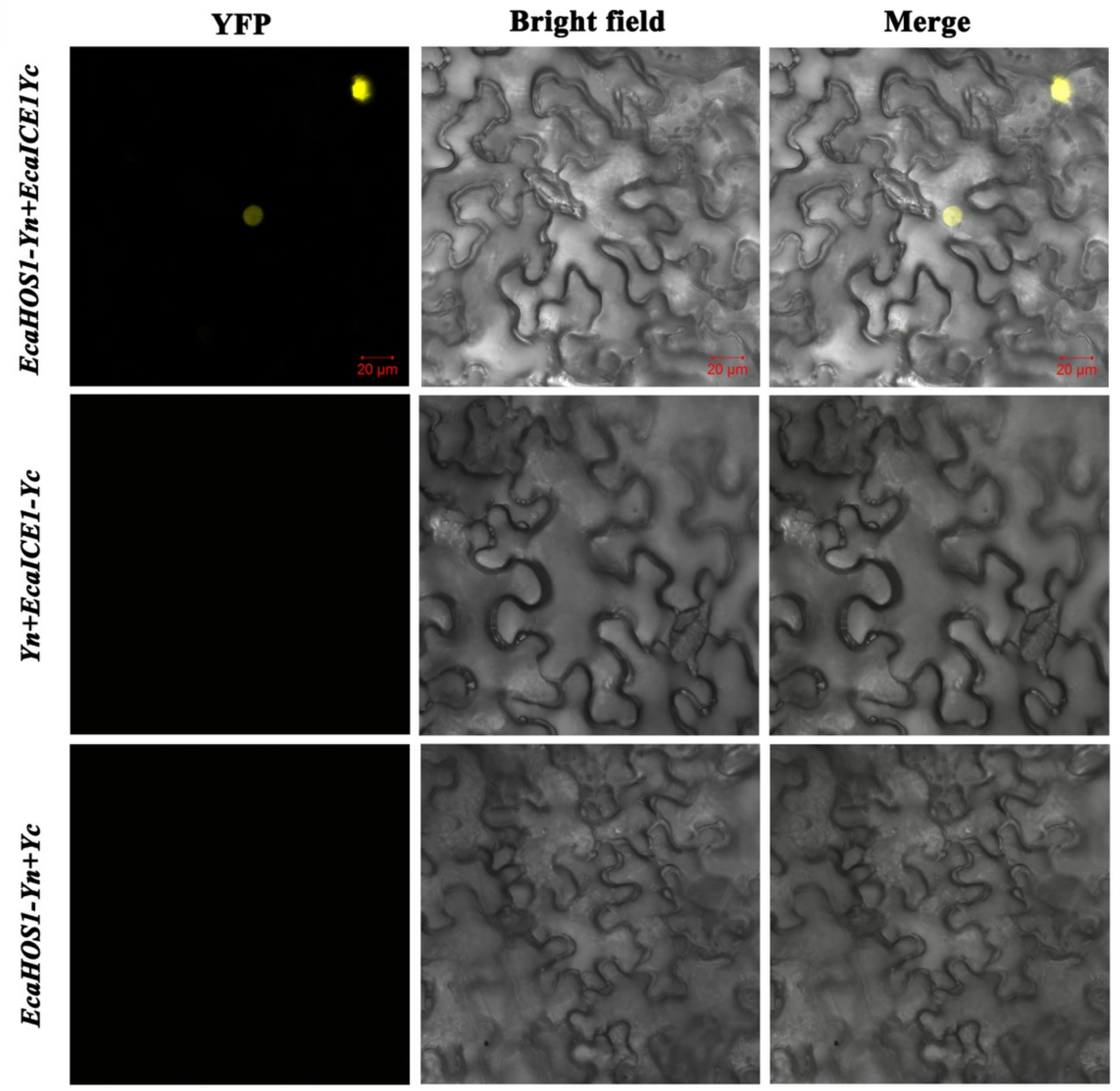

Figure 3

Bimolecular fluorescence complementation results of EcalCE1 and EcaHOS1 p35S::EcaHOS1Yn:p35S::EcalCE1-Yc were co-expressed in tobacco cells; p35s::Yn:p35S::EcalCE1-Yc and p35S::EcaHOS1-Yn:p35s::Yc served as negative controls. The first column is the dark field fluorescence signal plot, the second column is the bright field plot, and the third column is the dark field and bright field plot. Bar $=20 \mu \mathrm{m}$. 


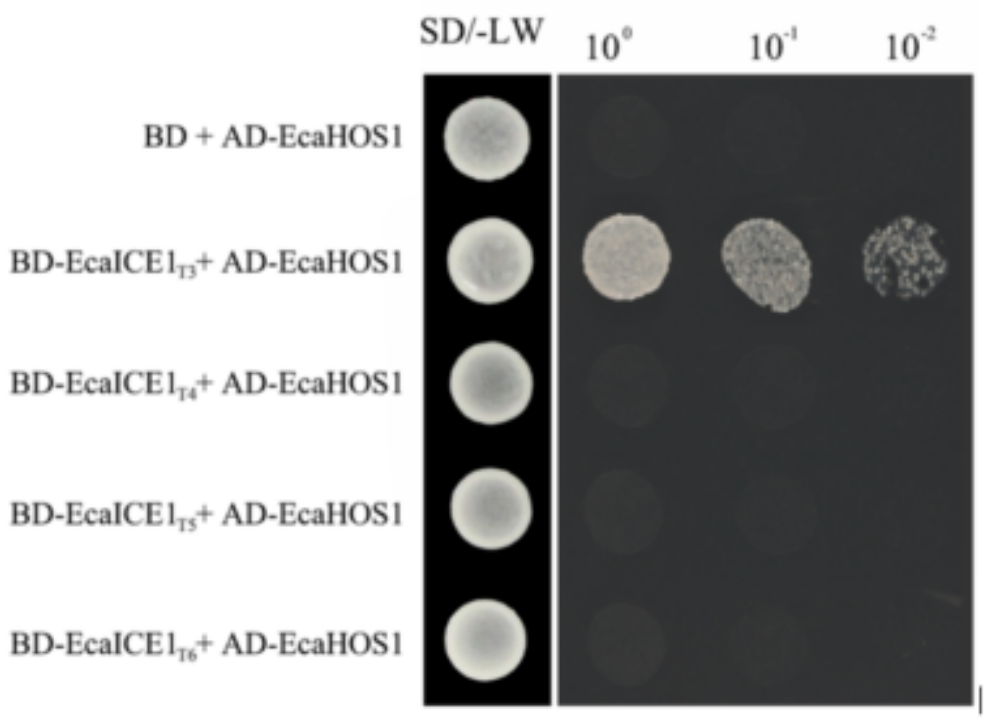

4B

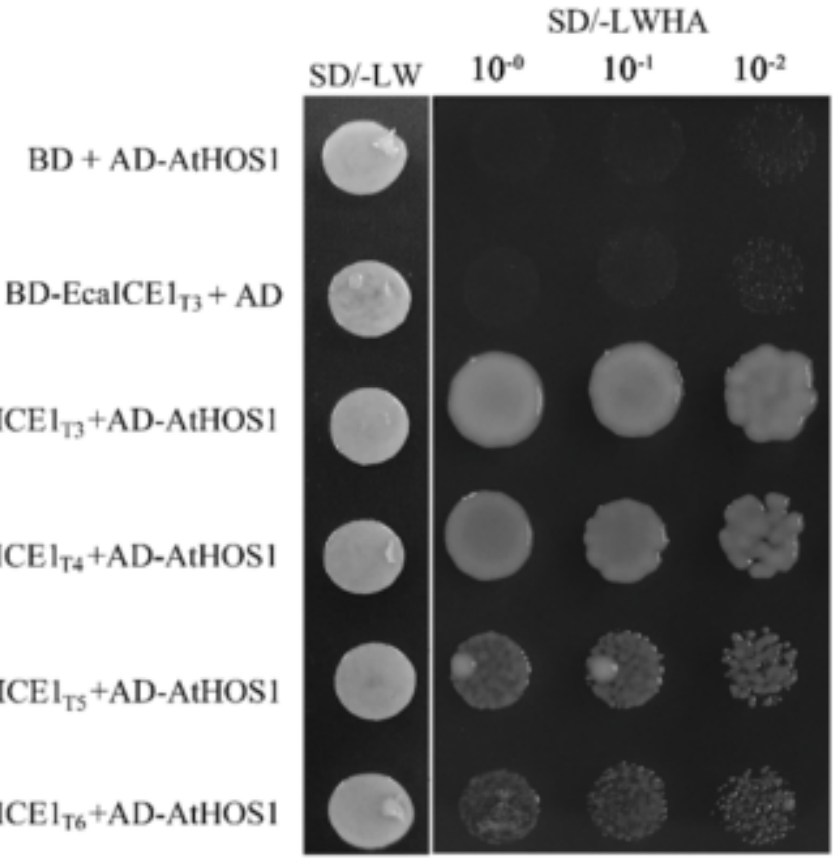

\section{Figure 4}

The interaction identification of truncated EcalCE1 with EcaHOS1 (4A) and AtHOS1 (4B) by yeast twohybrid methods BD: pGBKT7 vetor; AD: pGADT7 vetor; SD/-LW: SD/-Leu-Trp: double dropouts, SD medium with -Leu/-Trp; SD/-LWHA: quadruple dropouts, SD medium with-Leu-Trp-His-Ade; 100囚10-1ه10-2: dilutions with 1,10 and 100 times respectively. 
5 A

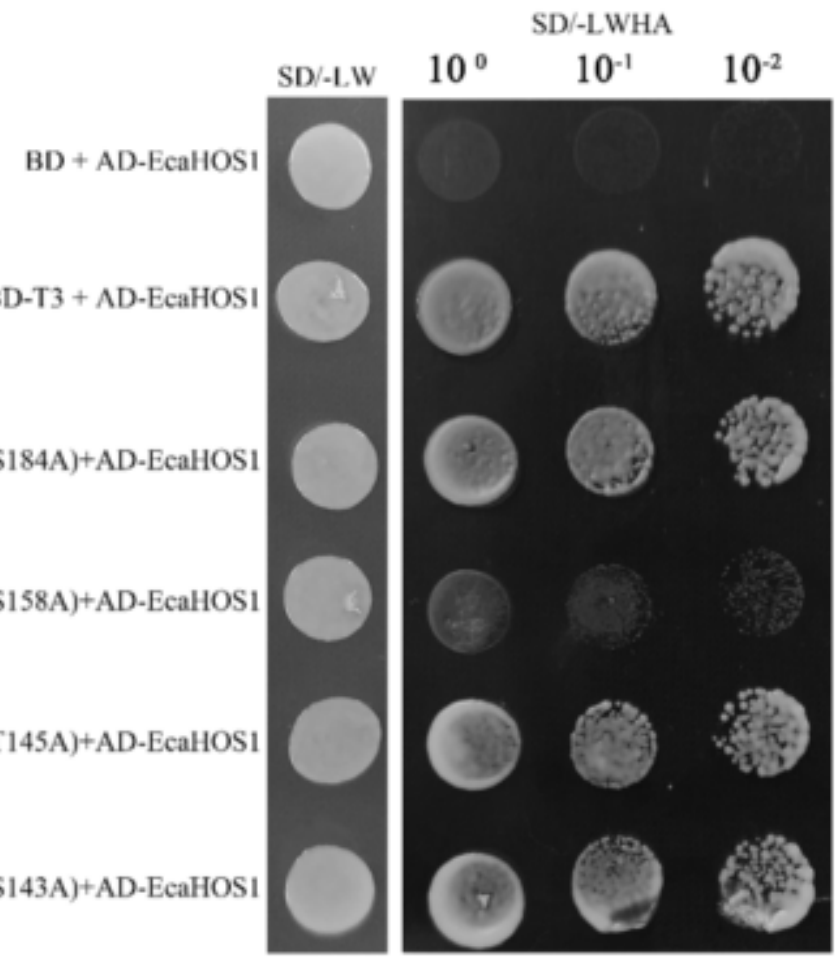

5B

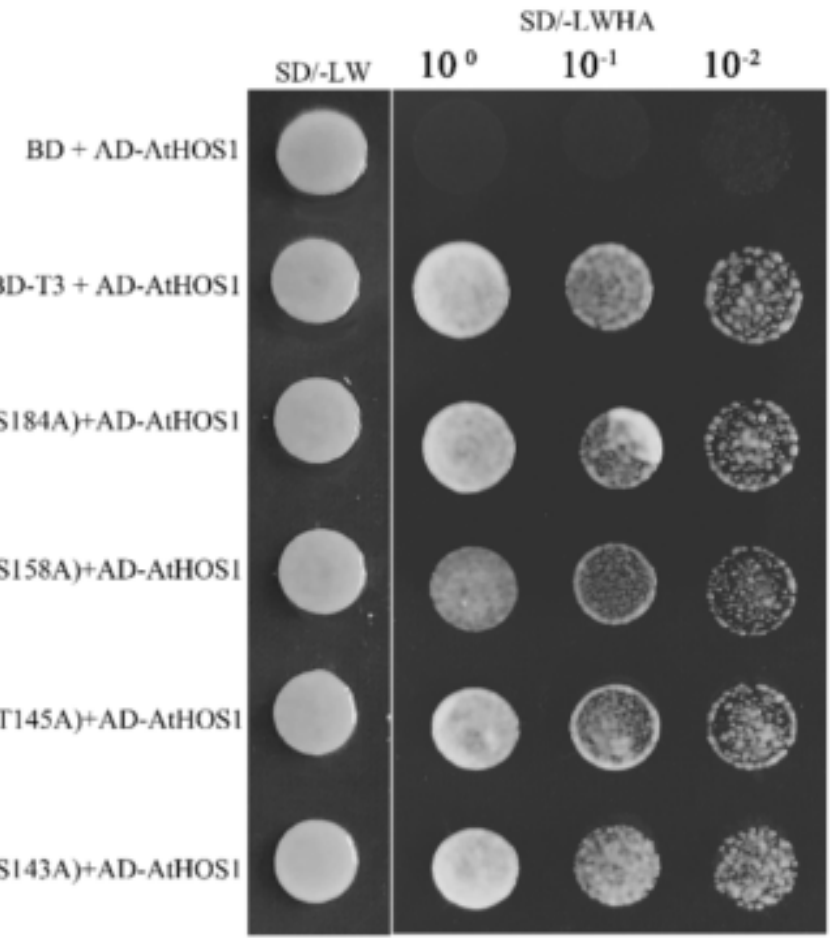

\section{Figure 5}

The interaction identification of EcalCE1-T3 and its mutants with EcaHOS1 (5A) and AtHOS1 (5B) by yeast two-hybrid methods BD: pGBKT7 vetor; AD: pGADT7 vetor; T3: truncated EcaICE1-T3; T3(S143A), T3(T145A), T3(S158A), T3(S184A): the mutant of truncated EcalCE1-T3 at serine 143, threonine 154, serine 158 and serine 184 by alanine, respectively; SD/-LW: SD/-Leu-Trp: double dropouts, SD medium 
with -Leu/-Trp; SD/-LWHA: quadruple dropouts, SD medium with-Leu-Trp-His-Ade; 100冈10-1ه10-2: dilutions with 1,10 and 100 times respectively.
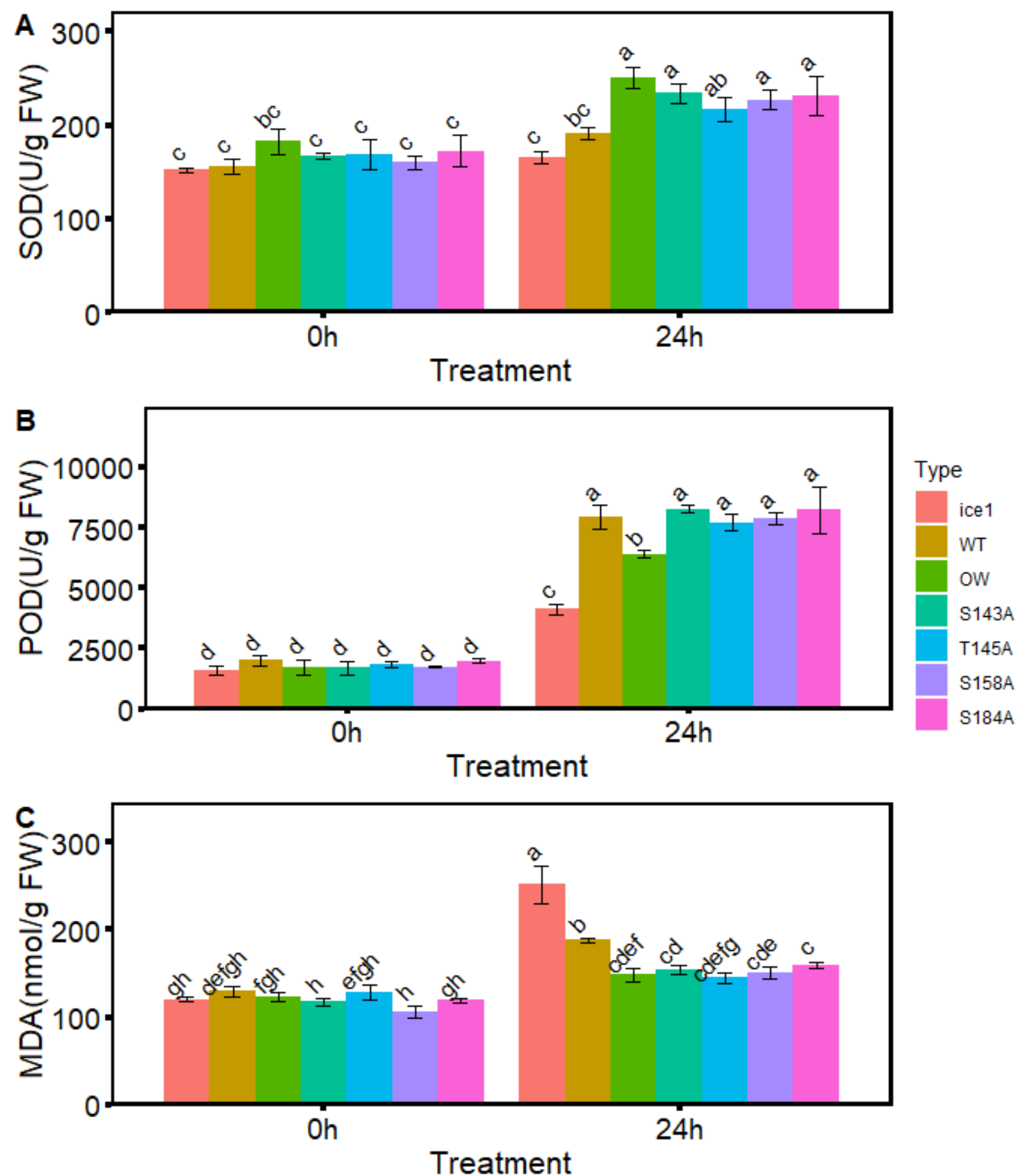

Figure 6

Biochemical Changes of different types of Arabidopsis plants under 4 oC for 0 h and $24 \mathrm{~h}$. A C: SOD activity, POD activity and MDA content of ice 1 mutant, wild type and transgenic Arabidopsis during cold treatment at $4 \mathrm{oC}$ for $0 \mathrm{~h}$ and 24h, respectively. ice1: ice1 mutant Arabidopsis; WT: wild-type Arabidopsis 
lines; OW: Arabidopsis lines over-expressing of EcalCE1; S143A, S145A, S158A, S184A: Arabidopsis lines over-expressing of EcalCE1 mutant at serine 143, threonine 154, serine 158 and serine 184 by alanine, respectively. Data are mean $\pm S E$ (standard error, $n=3$ ) with the same letter that are not significantly different at $a=0.05$ using Duncan's test.
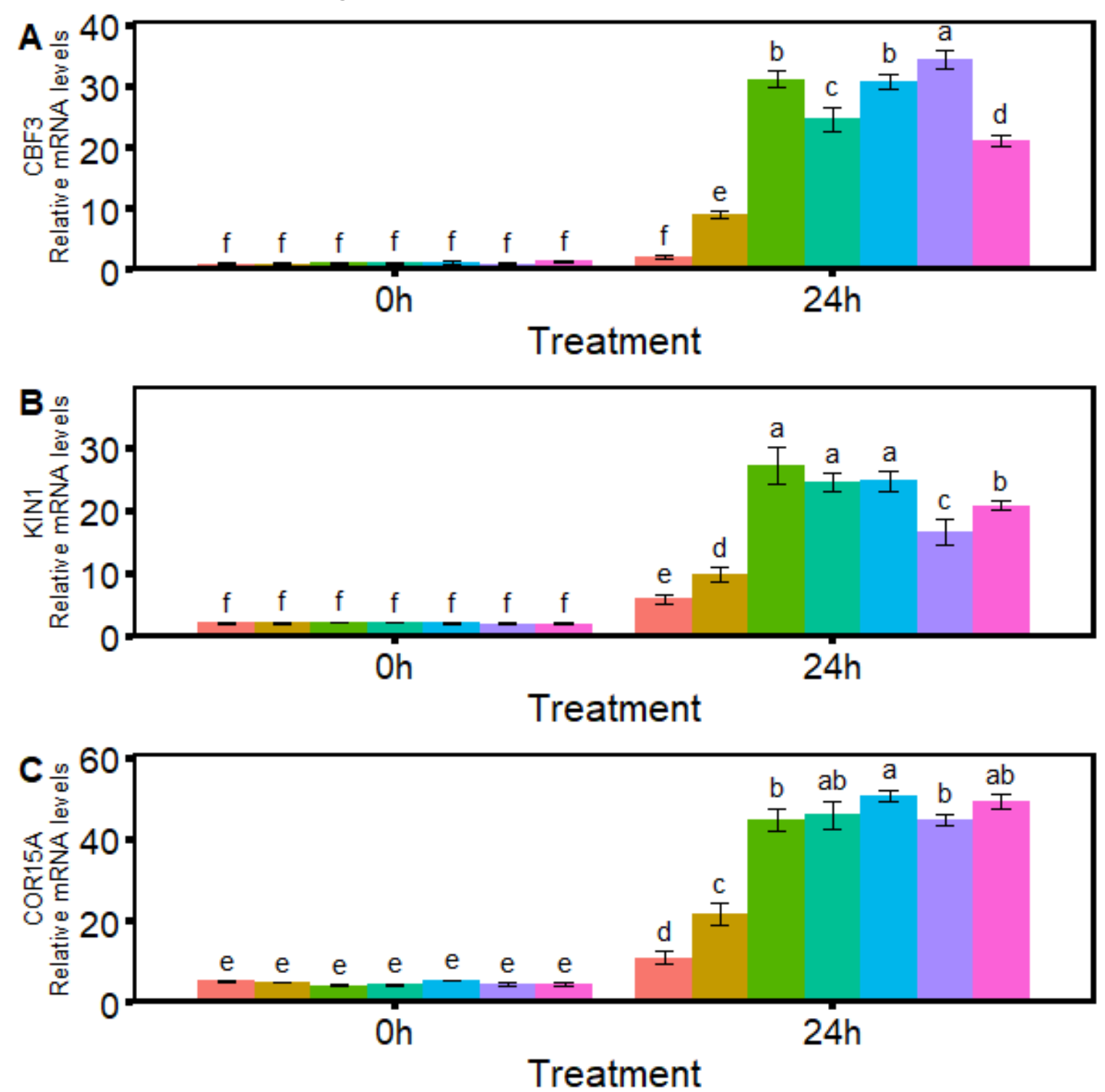

Type ice1 wt OW

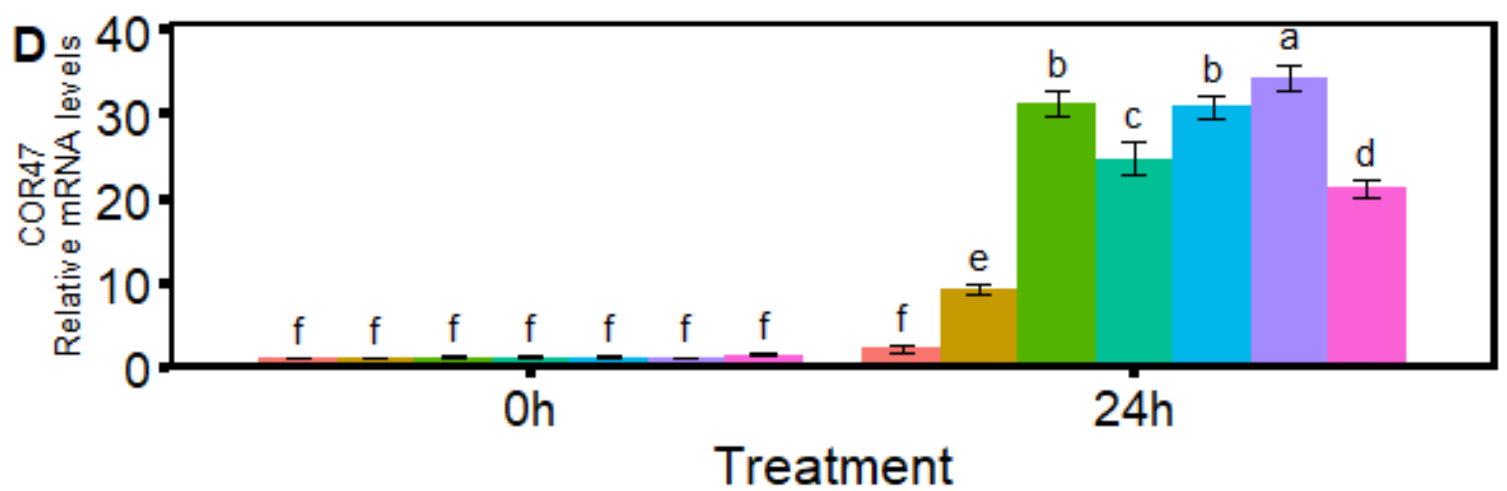

Figure 7 
Transcription patterns of cold-regulated genes in different types of Arabidopsis plants under $4 \mathrm{oC}$ for $0 \mathrm{~h}$ and 24h. A D: Relative mRNA transcript levels of transgenic ICE1, endogenous CBF3, KIN1, COR15 and COR47 in ice 1 mutant, wild type and transgenic Arabidopsis during cold treatment at $4 \mathrm{oC}$ for $0 \mathrm{~h}$ and $24 \mathrm{~h}$, respectively. ice1: ice1 mutant Arabidopsis; WT: wild-type Arabidopsis lines; OW: Arabidopsis lines overexpressing of EcalCE1; S143A, S145A, S158A, S184A: Arabidopsis lines over-expressing of EcalCE1 mutant at serine 143 , threonine 154 , serine 158 and serine 184 by alanine, respectively. Data are mean \pm $\mathrm{SE}$ (standard error, $\mathrm{n}=3$ ) with the same letter that are not significantly different at $\mathrm{a}=0.05 \mathrm{using}$ Duncan's test.

\section{Supplementary Files}

This is a list of supplementary files associated with this preprint. Click to download.

- ChengSupportingInformation.docx 